\title{
Reducing Power Loss in Magnetic Bearings by Optimizing Current Allocation
}

\author{
Tingshu Hu, Senior Member, IEEE, Zongli Lin, Senior Member, IEEE, and Paul E. Allaire
}

\begin{abstract}
Conventional magnetic bearings control current or flux by operating symmetrically about a bias current or bias flux. This approach is known to be much easier to control than operating without a bias but has the disadvantage of introducing additional power loss. Although one obvious way to minimize the power loss is to alternate activation of the two opposing electromagnetic actuators, this single actuator allocation strategy has not been successful in practical applications because it results in severe performance degradation. In this paper, we investigate the fundamental reasons behind the performance degradation under the single actuator allocation strategy. One major reason is voltage saturation in the circuit systems. On the basis of this result, we formulate the problem of minimizing the energy consumption by allocating the currents under the constraint of bounded voltages. We establish necessary conditions and properties for the optimal solution, which we use to determine the optimal allocation strategy for some common force signals. Since the optimal solution is very sensitive to the variation of the force signal, we propose a simple static allocation strategy to approximate the optimal solution.
\end{abstract}

Index Terms-Feedback control, magnetic bearings, optimal current allocation, power loss.

\section{INTRODUCTION}

$\mathbf{M}$ AGNETIC bearings have several appealing advantages over traditional bearings, such as very low power loss, very long life, elimination of oil supply, low weight, reduction of oil supply fire hazard, vibration control, and diagnostic capability [1]. Even though the power loss in magnetic bearings is much lower than in traditional fluid film bearings, there is still a significant potential for its further reduction. Conventional magnetic bearings control current or flux by operating symmetrically about a bias current or bias flux. This approach is known to be much easier to control than operating without a bias but has the disadvantage of introducing additional power consumption. Much research has been carried out toward reducing the bias or the power consumption (see, e.g., [2], [4]-[6], [11], [12]). A simple way of reducing power consumption is to use permanent magnets to generate the bias. For better control performances, we may choose to use pairs of electromagnets to generate the bias. In this case, the obvious way to minimize the power loss is to activate only one of the pair of electromagnets

Manuscript received April 9, 2002; revised January 5, 2004. This work was supported in part by the National Science Foundation under Grant CMS-0324329.

T. Hu and Z. Lin are with the Department of Electrical and Computer Engineering, University of Virginia, Charlottesville, VA 22904-4743 USA (e-mail: th7f@virginia.edu; z15y@ virginia.edu).

P. E. Allaire is with the Department of Mechanical and Aerospace Engineering, University of Virginia, Charlottesville, VA 22904-4743 USA (e-mail pea@virginia.edu).

Digital Object Identifier 10.1109/TMAG.2004.826613 at a time. This single actuator allocation strategy has not been successful in practical applications because it results in severe performance degradation. The objective of this paper is to find a way to minimize the power loss while certain system performances are maintained.

Our study will be based on a simple model which captures the fundamental features of magnetic bearing systems. Consider a rotor whose one-dimensional position is controlled by a pair of opposing electromagnets. The dynamics of the rotor can be typically modeled by the following differential equation (see, e.g., [3], [7]):

$$
M \ddot{x}=-B \dot{x}+F_{2}-F_{1}+d
$$

where $x$ is the position (or the angular displacement) and $M$ is the mass (or the moment of mass). $F_{1}$ and $F_{2}$ are the forces (or torques) produced by the two electromagnets and $d$ is the disturbance resulting from, for example, unbalance or aerodynamic loads. The basic requirement is to stabilize the rotor at the equilibrium point $(x, \dot{x})=(0,0)$ with net force $F_{n}:=F_{2}-F_{1}$. The forces $F_{1}$ and $F_{2}$ are generated by two electromagnetic circuits described by the following differential equations (see, e.g., [7]):

$$
\begin{aligned}
& L_{1} \dot{I}_{1}=v_{1}-I_{1} \frac{d L_{1}}{d x} \dot{x}-R_{1} I_{1} \\
& L_{2} \dot{I}_{2}=v_{2}-I_{2} \frac{d L_{2}}{d x} \dot{x}-R_{2} I_{2}
\end{aligned}
$$

where $L_{1}=\left(c_{\ell 1}\right) /\left(g_{0}+x\right)$ and $L_{2}=\left(c_{\ell 2}\right) /\left(g_{0}-x\right)$. The forces are determined from the air gap fluxes in terms of $I_{1}, I_{2}$ and $x$ as follows:

$$
F_{1}=c_{f 1}\left(\frac{g_{0} I_{1}}{g_{0}+x}\right)^{2}, \quad F_{2}=c_{f 2}\left(\frac{g_{0} I_{2}}{g_{0}-x}\right)^{2} .
$$

For simplicity, we assume in this paper that $c_{\ell 1}=c_{\ell 2}=$ $c_{\ell}, R_{1}=R_{2}=R$, and $c_{f 1}=c_{f 2}=c_{f}$.

Traditionally, there are two modes (or approaches) in the controlling of the above magnetic bearing system: the current mode and the voltage mode. In the current mode, the control inputs are the currents $I_{1}$ and $I_{2}$ and the voltages are ignored. And it is assumed that any desired currents $I_{1}$ and $I_{2}$ can be exactly produced without any delay and hence any desired net force $F_{n}$ can be exactly produced. In the voltage mode, the control inputs are the voltages $v_{1}$ and $v_{2}$, and all the dynamics of the power amplifier, the circuit constraint, etc., are taken into account. The control system model resulting from the current mode is much simpler than that from voltage mode. However, because of the ignored circuit dynamics, unpredicted performance degradation 
may occur during the operation of the practical system. The voltage mode results in an exact model but is more difficult for control design of real systems.

No matter which control mode is used, we have two objectives in the control design: to enhance system performances and to reduce power loss. Since the mechanical system (1) is very simple and easy to control if any desired force $F_{n}$ can be exactly generated (or if approximately generated, the error can be considered as a small disturbance), the system performances depend mainly on the tracking of a desired force signal by the actual force generated by the electromagnets.

Experience shows that the objective of tracking a desired force signal and that of reducing power loss are conflicting. For instance, in the current mode, there are infinitely many ways to allocate the two currents for a given $F_{n}$. Some allocations result in large power loss while others result in low power loss. It is obvious that the minimal power loss allocation strategy is to activate the two circuits alternatively, i.e., at least one of the currents $I_{1}$ and $I_{2}$ is zero at any time instant. However, this single actuator allocation usually results in poor performance. Another simple allocation strategy is to introduce a bias current $I_{b}$ and let $I_{1}=I_{b}+I$ and $I_{2}=I_{b}-I$, where $|I| \leq I_{b}$ for all time. This strategy results in good performances but has the disadvantage of introducing additional power loss.

To reach a good balance between these two objectives, we consider the problem of minimizing power loss while certain force tracking performance measures are maintained. As a first step, we need to identify the fundamental reason behind the performance degradation when the minimal power loss allocation strategy is used. We will find out in Section II that the main reason is that the single actuator allocation strategy may corrupt the assumption of exact following of a desired current signal by the one that is actually generated. This is in turn caused by the saturation of the voltages. This result indicates that we must take into account the saturation of the voltages in an effort to achieve our objective, and first of all, in formulating our objective into a meaningful mathematical optimization problem. In Section III, we formulate the problem of minimizing energy consumption under the constraint of voltage saturation and then establish the necessary conditions and some properties of the optimal solution. With these necessary conditions and properties, we can determine the exact optimal solution for some class of force signals. Since the optimal solution at a time instant depends on the future value of the force and is very sensitive to the change of the force signal, we will also propose a simple static allocation strategy in Section IV, which will reduce power loss to a level very close to the optimal one. Section V draws some concluding remarks.

\section{InVESTigation of Performance Degradation}

\section{A. Force Tracking Performance for a Closed-Loop System}

As we have discussed in the introduction, the mechanical system (1) is simple and easy to control, and the performances of the magnetic bearing system rely mainly on the performance of tracking a desired force signal. Hence, in this paper, we will focus on the force tracking performance. In the sequel, we will use $F_{n d}, I_{1 d}$, and $I_{2 d}$ to denote the desired force and current signals and use $F_{n}, I_{1}$ and $I_{2}$ to denote the actual force and currents.

The closed-loop design for the circuit systems (2)-(3) can be divided into two parts:

Part 1) Design a current allocation algorithm

$$
I_{1 d}=h_{1}\left(F_{n d}, x\right), \quad I_{2 d}=h_{2}\left(F_{n d}, x\right)
$$

such that

$$
c_{f}\left(\frac{g_{0} I_{2 d}}{g_{0}-x}\right)^{2}-c_{f}\left(\frac{g_{0} I_{1 d}}{g_{0}+x}\right)^{2}=F_{n d} .
$$

Part 2) Design control laws for each circuit system

$$
v_{1}=g_{1}\left(I_{1}, I_{1 d}, x, \dot{x}\right), \quad v_{2}=g_{2}\left(I_{2}, I_{2 d}, x, \dot{x}\right)
$$

such that the error between $I_{1}$ and $I_{1 d}$ and the error between $I_{2}$ and $I_{2 d}$ are sufficiently small.

As we can expect, the actual force $F_{n}$ generated by the above designed circuit systems would not be exactly the same as $F_{n d}$. One of our design objectives is to make the tracking error small by properly choosing the functions $h_{1}, h_{2}, g_{1}$, and $g_{2}$. The other objective is to minimize the power loss. It appears that these two objectives are independent: the force tracking performance only depends on the current tracking performance, which is determined by $g_{1}$ and $g_{2}$; and the power loss depends only on $h_{1}$ and $h_{2}$. But experience shows that these two objectives are conflicting. This section is dedicated to investigating the fundamental reason that causes the conflict.

\section{B. Observations on Performance Degradation}

In our study, we will use a sinusoidal force signal $F_{n d}$ to demonstrate the performance degradation. For simplicity, we consider the case where $(x, \dot{x})=(0,0)$. This is meaningful since it is the case when the system operates in its steady state and we are mainly concerned about the power loss at steady state. Let $L_{0}=c_{\ell} / g_{0}$. Then, the two circuit systems are

$$
L_{0} \dot{I}_{1}=v_{1}-R I_{1}, \quad L_{0} \dot{I}_{2}=v_{2}-R I_{2} .
$$

To track the desired current signals $I_{1 d}$ and $I_{2 d}$, we can use simple feedback laws $v_{1}=g_{1}\left(I_{1}, I_{1 d}\right)=k\left(I_{1 d}-I_{1}\right)$ and $v_{2}=g_{2}\left(I_{2}, I_{2 d}\right)=k\left(I_{2 d}-I_{2}\right)$. Ideally, if there is no bound on the voltage supply, then any periodic signal can be arbitrarily closely tracked by choosing a sufficiently large gain $k$. However, because of the actual bounds on $v_{1}$ and $v_{2}$, different current allocation strategies will result in different tracking performances when the same force signal $F_{n d}$ is desired. We will compare the following two allocation strategies.

1) Full Bias Allocation: Let $F_{m}$ be the magnitude of the sinusoidal signal $F_{n d}(t)$, then

$$
I_{2 d}=\frac{F_{m}+F_{n d}}{2\left(c_{f} F_{m}\right)^{\frac{1}{2}}}, \quad I_{1 d}=\frac{F_{m}-F_{n d}}{2\left(c_{f} F_{m}\right)^{\frac{1}{2}}} .
$$

In this case, both $I_{1 d}$ and $I_{2 d}$ are sinusoidal signals centered at $\left(F_{m} / c_{f}\right)^{(1 / 2)} / 2=: I_{b}$, the bias current. The minimum value of $I_{1 d}$ and $I_{2 d}$ is 0 and their maximum value is $\left(F_{m} / c_{f}\right)^{(1 / 2)}$. 

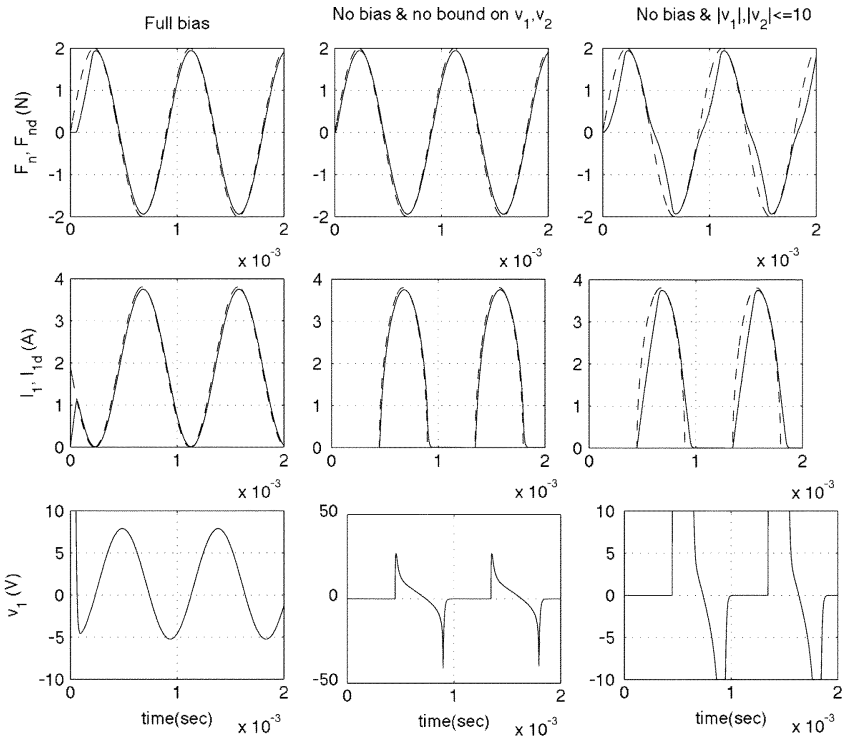

Fig. 1. Reason for bad tracking: voltage saturation and improper current allocation.

2) Single Actuator Allocation: In this allocation strategy, at least one of $I_{1 d}$ and $I_{2 d}$ is zero at any time, i.e., $I_{1 d} I_{2 d} \equiv 0$

$$
I_{1 d}=\left\{\begin{array}{ll}
0, & \text { if } F_{n d} \geq 0 \\
\left(-F_{n d} / c_{f}\right)^{\frac{1}{2}}, & \text { if } F_{n d}<0
\end{array} .\right.
$$

and

$$
I_{2 d}=\left\{\begin{array}{ll}
\left(F_{n d} / c_{f}\right)^{\frac{1}{2}}, & \text { if } F_{n d} \geq 0 \\
0, & \text { if } F_{n d}<0
\end{array} .\right.
$$

We also call it alternative allocation or no bias allocation.

In the following example, we compare the force tracking performances under these two current allocation strategies.

Example 1: Consider the circuit system model of a beam balancing test rig [3]

$$
L_{0} \dot{I}_{1}=v_{1}-R I_{1}, \quad L_{0} \dot{I}_{2}=v_{2}-R I_{2}
$$

where $L_{0}=4.9060 \times 10^{-4} \mathrm{H}$ and $R=0.7 \Omega$. The coefficient of force is $c_{f}=0.1384 \mathrm{~N} / \mathrm{A}^{2}$ and the desired force signal is $F_{n d}(t)=2 \sin (7000 t) \mathrm{N}$. The voltage control laws are $v_{1}=k\left(I_{1 d}-I_{1}\right)=50\left(I_{1 d}-I_{1}\right)$ and $v_{2}=50\left(I_{2 d}-I_{2}\right)$. Fig. 1 illustrates the difference of tracking performances between the two allocation strategies. In Fig. 1, the first row plots the net force $F_{n}$ (in solid curves) and the desired signal $F_{n d}$ (in dashed curves), the second row plots the current $I_{1}$ (in solid curves), and the desired signal $I_{1 d}$ (in dashed curves) and the third row plots the voltage $v_{1}$. We do not plot $I_{2}, I_{2 d}$, and $v_{2}$ because they have the same shapes as $I_{1}, I_{1 d}$ and $v_{1}$ but only with different phases. In the first column are the simulation results for the full bias case and the voltages are bounded by $\left|v_{1}\right|,\left|v_{2}\right| \leq 10$. Actually, for this case, the bounds on the voltages have no effect since in the steady state, both $v_{1}$ and $v_{2}$ are within the bound. The second column shows the simulation results for the case of single actuator allocation with no bounds on the voltages. The third column shows the simulation results for the case of single actuator allocation with the voltages bounded by $10 \mathrm{~V}$. By comparing the simulation results, we see that if there are no bounds on the voltages, then there is no obvious difference on the tracking of the

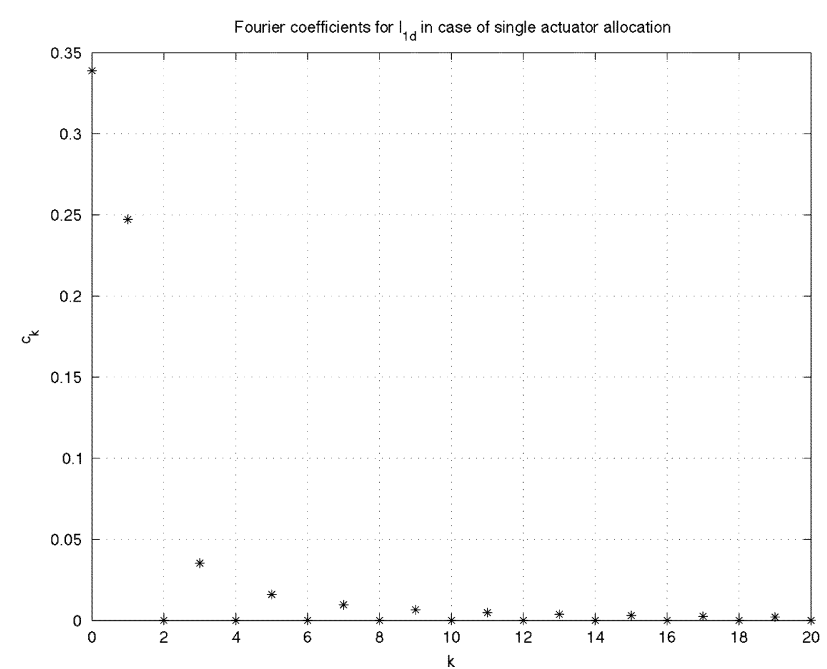

Fig. 2. Fourier coefficients of $I_{1 d}$ in case of single actuator allocation.

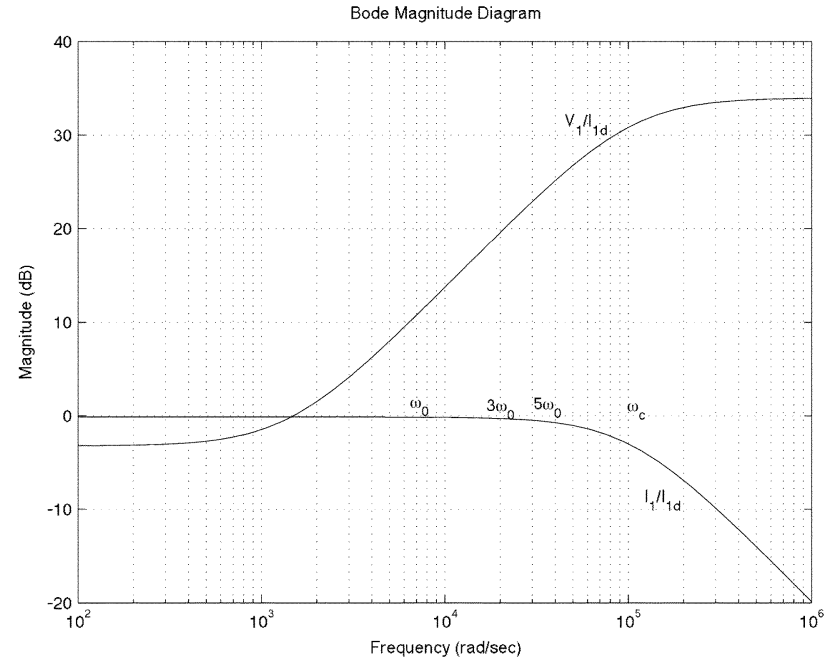

Fig. 3. Bode plot of $\left(I_{1}\right) /\left(I_{1 d}\right)$ and $\left(V_{1}\right) /\left(I_{1 d}\right)$.

desired net force between the two allocation strategies. The bad tracking performance in the third column is a result of the combined effects of the voltage bound and the allocation strategy.

\section{Explanation Using Bode Plot}

Given a sinusoidal signal $F_{n d}$, different current allocations will result in different shapes of the desired current signals $I_{1 d}$ and $I_{2 d}$. In the case of full bias allocation, $I_{1 d}$ is also a sinusoidal signal. In the case of single actuator allocation, $I_{1 d}$ is periodic but has some higher frequency components. The coefficients $c_{k}$ of the frequency components are plotted in Fig. 2 for $k=$ $0,1,2, \ldots, 20$.

Let us use the circuit systems in Example 1 to explain the effect of the higher frequency terms on the tracking performance of the currents. The transfer function from $I_{1 d}$ to $I_{1}$ and that from $I_{1 d}$ to $v_{1}$ are

$$
\frac{I_{1}(s)}{I_{1 d}(s)}=\frac{k}{L_{0} s+k+R}, \quad \frac{V_{1}(s)}{I_{1 d}(s)}=\frac{k\left(L_{0} s+R\right)}{L_{0} s+k+R} .
$$

The Bode plots of these transfer functions are shown in Fig. 3. The cutoff frequency of $I_{1} / I_{1 d}$ is $\omega_{c}=1.0334 \times 10^{5} \mathrm{rad} / \mathrm{s}$. 
In Example 1, the frequency of $F_{n d}$ and $I_{1 d}$ is $\omega_{0}=7000 \mathrm{rad} / \mathrm{s}$. In the case of single actuator allocation, we have $n \omega_{0} \leq \omega_{c}$ for $n \leq 13$. Hence, all the main frequency components of $I_{1 d}$ are kept in $I_{1}$ if there are no bounds on the voltages. This explains the close tracking of $I_{1 d}$ by $I_{1}$ in the second column of Fig. 1. However, the higher frequency terms do impose a larger requirement on the voltage $v_{1}$, as can be seen from the Bode plot of $V_{1} / I_{1 d}$. The magnitude of $V_{1} / I_{1 d}$ is about $10 \mathrm{~dB}$ at $\omega_{0}, 20 \mathrm{~dB}$ at $3 \omega_{0}$, and $25 \mathrm{~dB}$ at $5 \omega_{0}$. This explains why $v_{1}$ exceeds the upper and lower bounds of $10 \mathrm{~V}$ in the second column of Fig. 1. In the presence of voltage saturation, these high-frequency terms will cause the degradation of the current tracking performance, and consequently, the degradation of force tracking performance as demonstrated in the third column of Fig. 1.

\section{Interpretation in Terms of Slew Rate}

From the first small figure in the third column of Fig. 1, we observe that there is an obvious difference between the slew rates of $F_{n}$ and $F_{n d}$ in a neighborhood of $F_{n d}=0$. This corresponds to the big difference between $I_{1}$ and $I_{1 d}$ at the rising edges and the falling edges of $I_{1 d}$. At the rising edge, $I_{1 d}$ has a slope equal to infinity, which requires an infinite value of the voltage. The same thing happens at the falling edges.

Note that for the sinusoidal signal $F_{n d}$, the largest slew rate occurs at $F_{n d}=0$. For $F_{n}$, we have

$$
\dot{F}_{n}=2 c_{f}\left(I_{2} \dot{I}_{2}-I_{1} \dot{I}_{1}\right) \text {. }
$$

In the ideal situation, at $F_{n d}=0$, we have $I_{1}=I_{1 d}=I_{2}=$ $I_{2 d}=0$, since both $I_{1}$ and $I_{2}$ have infinite slope, the slew rate of $F_{n}$ can still be guaranteed. In real life, we also have $I_{1}=0$, but due to the bound on $v_{1}, \dot{I}_{1}$ is bounded. Hence, there is no contribution to the slew rate of $F_{n}$ from $I_{1}$. As to $I_{2}$, although nonzero at $F_{n}=0$, its contribution to the total force slew rate is also less than the desired value.

In view of the above arguments, an indirect way to improve the tracking performance is to ensure certain slew rate at $F_{n}=0$.

\section{POWER-LOSS REDUCTION BY OPTIMIZING CURRENT ALLOCATION}

The analysis results from last section indicate that voltage saturation is a key factor causing performance degradation. Therefore, in order to achieve the objective of minimizing power loss while maintaining a desired force tracking performance, we must take into account the bounds on the voltages.

\section{A. Open-Loop Optimization Problem}

In control theory, many fundamental problems are formulated in terms of open-loop control, such as controllability, time-optimal control, optimal tracking, and linear quadratic regulation. By open-loop control, we mean that the control signal in the whole time interval of interest is determined off-line. Although open-loop control has a lot of disadvantages and is rarely implemented in practical systems, it provides a limit to the performance that can be achieved with any kind of closed-loop control and it also provides us with a guideline for approximating the limit using closed-loop control. In this section, we will first for- mulate the problem of minimizing power loss into an open-loop problem. To do this, we need to assume full knowledge of the desired force signal $F_{n d}$ in certain time interval, say, $[0, T]$.

We will consider the case where $(x, \dot{x})=(0,0)$. As we have mentioned earlier, this is simple and also meaningful since we are mainly concerned about the power loss in the steady state. To keep the mechanical system (1) in the steady state, we need to use $F_{n}$ to cancel the disturbance $d$, i.e., $F_{n}=-d$. In the open-loop design, all the signals $v_{1}, v_{2}, I_{1}$, and $I_{2}$ are determined off-line such that the desired force $F_{n}$ is exactly produced. With all the above assumptions, the relation between the force and the currents is

$$
F_{n}=F_{2}-F_{1}=c_{f}\left(I_{2}^{2}-I_{1}^{2}\right) .
$$

Given a desired force signal $F_{n}(t) \in[0, T]$, our objective is to generate two current signals $I_{1}(t)$ and $I_{2}(t)$ to satisfy $F_{n}(t)=$ $c_{f}\left(I_{2}^{2}(t)-I_{1}^{2}(t)\right)$ and to minimize the total energy during the time interval $[0, T]$,

$$
E=c_{e} \int_{0}^{T}\left(I_{2}^{2}+I_{1}^{2}\right) d t
$$

where $c_{e}$ is some constant of the magnetic bearing system. The currents are generated by the circuit systems

$$
L_{0} \dot{I}_{1}=v_{1}-R I_{1}, \quad L_{0} \dot{I}_{2}=v_{2}-R I_{2}
$$

subject to the bounds on the voltages $\left|v_{1}\right|,\left|v_{2}\right| \leq v_{m}$. Assume that the initial conditions of the circuit systems, $I_{1}(0)$ and $I_{2}(0)$, are free to choose. This is reasonable since $T$ is usually much greater than the transient period and we can take any measure to bring the initial conditions to the desired values (the energy consumption during this transient period can be neglected). The energy minimization problem subject to the constraint of bounded voltages can be formulated as follows:

$$
\begin{array}{ll} 
& \min _{v_{1}, v_{2}, I_{1}(0), I_{2}(0)} \int_{0}^{T}\left(I_{1}^{2}+I_{2}^{2}\right) d t \\
\text { s.t. } & \text { a) } L_{0} \dot{I}_{1}=v_{1}-R I_{1} \\
& \text { b) } L_{0} \dot{I}_{2}=v_{2}-R I_{2} \\
\text { c) } c_{f}\left(I_{2}^{2}-I_{1}^{2}\right)=F_{n} \\
\text { d) }\left|v_{1}\right| \leq v_{m},\left|v_{2}\right| \leq v_{m} .
\end{array}
$$

Here, we note that $I_{1}$ and $I_{2}$ are intermediate variables depending on $v_{1}, v_{2}, I_{1}(0)$, and $I_{2}(0)$ from a) and $\mathrm{b}$ ).

\section{B. Conditions for the Optimal Solution}

To solve (5), we introduce five auxiliary scalar functions $\lambda(t), \alpha_{1}(t), \alpha_{2}(t), s_{1}(t)$, and $s_{2}(t), t \in[0, T]$, and form the Hamiltonian function

$$
\begin{aligned}
H=I_{1}^{2}+I_{2}^{2}+ & \lambda\left(I_{2}^{2}-I_{1}^{2}-F_{n} / c_{f}\right) \\
& +\alpha_{1}\left(v_{1}^{2}+s_{1}^{2}-v_{m}^{2}\right)+\alpha_{2}\left(v_{2}^{2}+s_{2}^{2}-v_{m}^{2}\right)
\end{aligned}
$$

The last three terms deal with the constraints c) and d). As to constraints a) and b), they can be relaxed with

$$
I_{1}(t)=e^{-R t / L_{0}} I_{1}(0)+\frac{1}{L_{0}} \int_{0}^{t} e^{-R(t-\tau) / L_{0}} v_{1}(\tau) d \tau
$$


and

$$
I_{2}(t)=e^{-R t / L_{0}} I_{2}(0)+\frac{1}{L_{0}} \int_{0}^{t} e^{-R(t-\tau) / L_{0}} v_{2}(\tau) d \tau
$$

respectively. We note from the above equations that $I_{1}(t)$ and $I_{2}(t)$ are continuous signals under the constraint of bounded voltages.

Define

$$
\Phi\left(v_{1}, v_{2}, I_{1}(0), I_{2}(0), \lambda, \alpha_{1}, \alpha_{2}, s_{1}, s_{2}\right):=\int_{0}^{T} H d t .
$$

We assume that all the variables $v_{1}, v_{2}, \lambda, \alpha_{1}, \alpha_{2}, s_{1}$, and $s_{2}$ are piecewise continuous functions.

Suppose that $\left(v_{1}^{*}, v_{2}^{*}, I_{1}^{*}(0), I_{2}^{*}(0)\right)$ is an optimal solution to (5), then there exist $\lambda^{*}, \alpha_{1}^{*}, \alpha_{2}^{*}, s_{1}^{*}$, and $s_{2}^{*}$ which jointly result in a stationary point of $\Phi$, where the variation of $\Phi$ with respect to each variable is zero. Let us use the variation of $\Phi$ with respect to $v_{1}$ to illustrate the idea. Let $\Delta v_{1}$ be a perturbation direction of $v_{1}$ and consider $v_{1}=v_{1}^{*}+\varepsilon \Delta v_{1}$ for arbitrarily small $\varepsilon$. Define

$$
\begin{aligned}
J_{v_{1}}\left(\varepsilon, \Delta v_{1}\right):=\Phi\left(v_{1}^{*}+\varepsilon \Delta v_{1}, v_{2}^{*},\right. & I_{1}^{*}(0), \\
& \left.I_{2}^{*}(0), \lambda^{*}, \alpha_{1}^{*}, \alpha_{2}^{*}, s_{1}^{*}, s_{2}^{*}\right) .
\end{aligned}
$$

The variation of $\Phi$ with respect to $v_{1}$ along the direction $\Delta v_{1}$ is defined as

$$
\left.\frac{\partial J_{v_{1}}}{\partial \varepsilon}\right|_{\varepsilon=0} .
$$

The stationary property for the optimal solution requires that

$$
\left.\frac{\partial J_{v_{1}}}{\partial \varepsilon}\right|_{\varepsilon=0}=0 \quad \forall \Delta v_{1} .
$$

In what follows, we transfer the above requirement to conditions on the optimal solution.

Because of the linearity of the circuit systems, under $v_{1}=$ $v_{1}^{*}+\varepsilon \Delta v_{1}$, we have $I_{1}=I_{1}^{*}+\varepsilon \Delta I_{1}$, where

$$
\Delta I_{1}(t)=\frac{1}{L_{0}} \int_{0}^{t} e^{-R(t-\tau) / L_{0}} \Delta v_{1}(\tau) d \tau
$$

It follows that

$$
\begin{aligned}
&\left.\frac{\left.\partial J_{v_{1}}\right|_{\varepsilon=0}=}{\partial \varepsilon}\right|_{0} ^{T}\left(1-\lambda^{*}(t)\right) I_{1}^{*}(t) \Delta I_{1}(t) d t \\
&+2 \int_{0}^{T} \alpha_{1}^{*}(t) v_{1}^{*}(t) \Delta v_{1}(t) d t \\
&= 2 \int_{0}^{T}\left(1-\lambda^{*}(t)\right) I_{1}^{*}(t) \frac{1}{L_{0}} \\
& \times \int_{0}^{t} e^{-R(t-\tau) / L_{0}} \Delta v_{1}(\tau) d \tau d t \\
&+2 \int_{0}^{T} \alpha_{1}^{*}(t) v_{1}^{*}(t) \Delta v_{1}(t) d t \\
&= 2 \int_{0}^{T} \frac{1}{L_{0}} e^{R \tau / L_{0}} \Delta v_{1}(\tau) \\
& \times \int_{\tau}^{T}\left(1-\lambda^{*}(t)\right) I_{1}^{*}(t) e^{-R t / L_{0}} d t d \tau \\
&+2 \int_{0}^{T} \alpha_{1}^{*}(t) v_{1}^{*}(t) \Delta v_{1}(t) d t
\end{aligned}
$$

$$
\begin{aligned}
= & 2 \int_{0}^{T} \frac{1}{L_{0}} e^{R t / L_{0}} \Delta v_{1}(t) \\
& \times \int_{t}^{T}\left(1-\lambda^{*}(\tau)\right) I_{1}^{*}(\tau) e^{-R \tau / L_{0}} d \tau d t \\
& +2 \int_{0}^{T} \alpha_{1}^{*}(t) v_{1}^{*}(t) \Delta v_{1}(t) d t \\
= & 2 \int_{0}^{T} \Delta v_{1}(t) \\
\times & \frac{1}{L_{0}} e^{R t / L_{0}} \int_{t}^{T}\left(1-\lambda^{*}(\tau)\right) I_{1}^{*}(\tau) e^{-R \tau / L_{0}} d \tau \\
& \left.+\alpha_{1}^{*}(t) v_{1}^{*}(t)\right) d t
\end{aligned}
$$

From (7) and (6), we have

$$
\frac{1}{L_{0}} e^{\frac{R t}{L_{0}}} \int_{t}^{T}\left(1-\lambda^{*}(\tau)\right) I_{1}^{*}(\tau) e^{-\frac{R \tau}{L_{0}}} d \tau+\alpha_{1}^{*}(t) v_{1}^{*}(t) \equiv 0 .
$$

Similar analysis on the variation of $\Phi$ with respect to other variables results in

$$
\begin{aligned}
& \frac{1}{L_{0}} e^{\frac{R t}{L_{0}}} \int_{t}^{T}\left(1+\lambda^{*}(\tau)\right) I_{2}^{*}(\tau) e^{-\frac{R \tau}{L_{0}}} d \tau+\alpha_{2}^{*}(t) v_{2}^{*}(t) \equiv 0 \\
& \int_{0}^{T}\left(1-\lambda^{*}(\tau)\right) I_{1}^{*}(\tau) e^{-\frac{R \tau}{L_{0}}} d \tau \equiv 0 \\
& \int_{0}^{T}\left(1+\lambda^{*}(\tau)\right) I_{2}^{*}(\tau) e^{-\frac{R \tau}{L_{0}}} d \tau \equiv 0 \\
& \alpha_{1}^{*}(t) s_{1}^{*}(t) \equiv 0 \\
& \alpha_{2}^{*}(t) s_{2}^{*}(t) \equiv 0 \\
& \left(I_{2}^{*}(t)\right)^{2}-\left(I_{1}^{*}(t)\right)^{2} \equiv F_{n}(t) / c_{f} \\
& \left(v_{1}^{*}(t)\right)^{2}+\left(s_{1}^{*}(t)\right)^{2} \equiv v_{m}^{2} \\
& \left(v_{2}^{*}(t)\right)^{2}+\left(s_{2}^{*}(t)\right)^{2} \equiv v_{m}^{2}
\end{aligned}
$$

For simplicity of analysis, we make the following weak assumptions on $F_{n}$ to exclude some special patterns in $F_{n}$.

Assumption 1:

i) $F_{n}(t)$ is continuously differentiable in $(0, T)$ and not identically zero in any subinterval of $[0, T]$.

ii) Let $I_{a}=\left(\left|F_{n}\right| / c_{f}\right)^{1 / 2}$. There is no interval $\left[t_{1}, t_{2}\right] \subset$ $[0, T]$ such that for all $t \in\left[t_{1}, t_{2}\right]$

$$
R I_{a}(t)+L_{0} \dot{I}_{a}(t)=v_{m}\left(\text { or }-v_{m}\right) .
$$

iii) There is no interval $\left[t_{1}, t_{2}\right] \subset[0, T]$, where there exist $I_{1}$ and $I_{2}$ such that for all $t \in\left[t_{1}, t_{2}\right]$

$$
I_{2}^{2}(t)-I_{1}^{2}(t)=F_{n}(t) / c_{f}
$$

and

$$
R I_{1}(t)+L_{0} \dot{I}_{1}(t)= \pm v_{m}, \quad R I_{2}(t)+L_{0} \dot{I}_{2}(t)= \pm v_{m}
$$

Without loss of generality, we also assume that $I_{1}(t), I_{2}(t) \geq$ 0 for all $t$. Otherwise, we can reverse the sign of the voltage when $I_{1}(t)$ or $I_{2}(t)$ starts to decrease from 0 .

Based on the stationary condition (8)-(16), and since all the signals are piecewise continuous, the interval $[0, T]$ can be divided into subintervals $\left(t_{i}, t_{i+1}\right)$, where all the optimal signals are continuous, of the following three types: 
- $\left|v_{1}^{*}(t)\right|,\left|v_{2}^{*}(t)\right|<v_{m}$ for all $t \in\left(t_{i}, t_{i+1}\right)$. Then, $s_{1}^{*}(t), s_{2}^{*}(t) \neq 0$ for all $t \in\left(t_{i}, t_{i+1}\right)$, and we must have $\alpha_{1}^{*}(t) \equiv \alpha_{2}^{*}(t) \equiv 0$ by (12) and (13). From (8) and (9), we obtain $\left(1-\lambda^{*}(t)\right) I_{1}^{*}(t) \equiv 0$ and $(1+$ $\left.\lambda^{*}(t)\right) I_{2}^{*}(t) \equiv 0$. Since $1-\lambda^{*}(t)$ and $1+\lambda^{*}(t)$ cannot be both zero, we must have $I_{1}^{*}(t) \equiv 0$ or $I_{2}^{*}(t) \equiv 0$ and $\lambda^{*}(t) \equiv-1$ or 1 .

- $\left|v_{1}^{*}(t)\right|=v_{m},\left|v_{2}^{*}(t)\right|<v_{m}$ for all $t \in\left(t_{i}, t_{i+1}\right)$. Then, similar to the first type, $s_{2}^{*}(t) \neq 0, \alpha_{2}^{*}(t) \equiv 0$ and $\left(1+\lambda^{*}(t)\right) I_{2}^{*}(t) \equiv 0$. Since $v_{1}^{*}(t) \equiv \pm v_{m}$, it is impossible to have $I_{2}^{*}(t) \equiv 0$ (which means that $\left.I_{1}=I_{a}\right)$ in any subinterval of $\left(t_{i}, t_{i+1}\right)$ by Assumption 1. Hence in this case, both $I_{1}^{*}(t)$ and $I_{2}^{*}(t)$ are nonzero and $\lambda^{*}(t) \equiv-1$.

- $\quad\left|v_{1}^{*}(t)\right|<v_{m},\left|v_{2}^{*}(t)\right|=v_{m}$ for all $t \in\left(t_{i}, t_{i+1}\right)$. This is similar to the second type. We have both $I_{1}^{*}(t)$ and $I_{2}^{*}(t)$ nonzero and $\lambda^{*}(t) \equiv 1$.

Note that we do not consider the possibility that $\left|v_{1}^{*}(t)\right| \equiv$ $v_{m},\left|v_{2}^{*}(t)\right| \equiv v_{m}$ in any subinterval. This could only happen for very special $F_{n}(t)$ that satisfies (18) and (19). By rearranging the above types of intervals, we can also obtain a classification of interval types in terms of currents. For clarity and future reference, we put this classification in the following:

\begin{tabular}{c|c|c|c|c|c|c|c}
\hline Type & $I_{1}^{*}$ & $I_{2}^{*}$ & $\left|v_{1}^{*}\right|$ & $\left|v_{2}^{*}\right|$ & $\lambda^{*}$ & $\alpha_{1}^{*}$ & $\alpha_{2}^{*}$ \\
\hline \hline $\mathrm{A}$ & 0 & $\neq 0$ & $<v_{m}$ & $<v_{m}$ & -1 & 0 & 0 \\
\hline $\mathrm{B}$ & $\neq 0$ & 0 & $<v_{m}$ & $<v_{m}$ & 1 & 0 & 0 \\
\hline $\mathrm{C}$ & $\neq 0$ & $\neq 0$ & $v_{m}$ & $<v_{m}$ & -1 & $\neq 0$ & 0 \\
\hline $\mathrm{D}$ & $\neq 0$ & $\neq 0$ & $<v_{m}$ & $v_{m}$ & 1 & 0 & $\neq 0$ \\
\hline
\end{tabular}

The above table means that if both $\left|v_{1}\right|$ and $\left|v_{2}\right|$ are strictly less than $v_{m}$, then one of the currents must be zero. On the other hand, if both of the currents are nonzero, then one of the voltages must take the maximum or the minimum value.

For the first two types, the currents are easy to determine. For Type $\mathrm{C}$ and Type $\mathrm{D}$, either $v_{1}^{*}= \pm v_{m}$ or $v_{2}^{*}= \pm v_{m}$. So the current $I_{i}^{*},(i=1$ or 2$)$, corresponding to $v_{i}^{*}= \pm v_{m}$, can be determined and the other one can be computed from $\left(I_{2}^{*}\right)^{2}-\left(I_{1}^{*}\right)^{2}=F_{n} / c_{f}$. The function $\lambda^{*}$ takes value 1 or -1 in every subinterval. As we will see later in an example, $\lambda^{*}$ may contain impulses at some instant $t_{i}$ where one type of interval is switched to another type. This results in a strictly nonzero weight on the term $I_{2}^{2}\left(t_{i}\right)-I_{1}^{2}\left(t_{i}\right)-F_{n}\left(t_{i}\right) / c_{f}$ in $\Phi$, indicating the special significance of switching instants.

For a general force signal $F_{n}$, the optimal allocated currents and the corresponding voltage signals usually contain different types of subintervals. Although the signals are easy to determine for each given type, it is not a simple task to find out how many subintervals are contained and to determine exactly the time instants where one type is switched to another type. The optimal signals would be practically impossible to determine if there are too many subintervals. Further information about the optimal signals can be revealed after more detailed examination. This information will help to simplify substantially the procedure of optimization. We will see that for a half cycle of a sinusoidal $F_{n}$, there are only three subintervals and we only need to search for a single switching instant to determine the optimal signals (the second switch depends on the first one).
Theorem 1: Let $\left(I_{1}^{*}, I_{2}^{*}, v_{1}^{*}, v_{2}^{*}\right)$ be a set of optimal signals. Suppose that the first interval is of Type A $\left(I_{1}^{*}=0, I_{2}^{*}>0\right)$. The signals can switch to Type B $\left(I_{1}^{*}>0, I_{2}^{*}=0\right)$ at $t=$ $t_{1}$ only if $F_{n}\left(t_{1}\right)=0$. They can switch to Type $\mathrm{D}\left(\left|v_{1}^{*}\right|<\right.$ $\left.v_{m}, v_{2}^{*}= \pm v_{m}\right)$ at $t=t_{1}$ only if $v_{2}^{*}\left(t_{1}^{-}\right)= \pm v_{m}$. Consider the case that they switch to Type $\mathrm{C}$ (with $v_{1}^{*}=v_{m},\left|v_{2}^{*}\right|<v_{m}$ ) at $t=t_{1}$. Assume that $\left|v_{2}^{*}\left(t_{1}\right)\right|<v_{m}$. Then, the signals will not switch to:

1) Type $\mathrm{D}$ before $\left|v_{2}^{*}\right|$ reaches $v_{m}$ or before $I_{2}^{*}$ reaches 0 ;

2) Type $\mathrm{C}$ (with $v_{1}^{*}=-v_{m},\left|v_{2}^{*}\right|<v_{m}$ ) before $\left|v_{2}^{*}\right|$ reaches $v_{m}$ or before $I_{2}^{*}$ reaches 0 ;

3) Type B before $I_{2}^{*}$ reaches 0 .

Remark 1: An optimal solution is expected to have Type A or Type B intervals. If $F_{n}(t)$ is periodic, then it does not matter when we start the signal and it is without loss of generality to assume that the optimal signals start with Type A interval.

Proof: The statement that the signals can switch to Type B $\left(I_{1}^{*}>0, I_{2}^{*}=0\right)$ at $t=t_{1}$ only if $F_{n}\left(t_{1}\right)=0$ is obvious since both $I_{1}^{*}$ and $I_{2}^{*}$ are continuous. For the next statement, let us start from the constraint $\left(I_{2}^{*}\right)^{2}-\left(I_{1}^{*}\right)^{2}=F_{n} / c_{f}$. Differentiating both sides, we have

$$
\begin{aligned}
& I_{2}^{*} \dot{I}_{2}^{*}-I_{1}^{*} \dot{I}_{1}^{*}=\dot{F}_{n} /\left(2 c_{f}\right), \\
& I_{2}^{*}\left(v_{2}^{*}-R I_{2}^{*}\right)-I_{1}^{*}\left(v_{1}^{*}-R I_{1}^{*}\right)=L_{0} \dot{F}_{n} /\left(2 c_{f}\right),
\end{aligned}
$$

and

$$
I_{2}^{*} v_{2}^{*}-I_{1}^{*} v_{1}^{*}=L_{0} \dot{F}_{n} /\left(2 c_{f}\right)+R F_{n} / c_{f}
$$

Suppose that there is a switch from Type A to Type D at $t=t_{1}$. Then, $I_{1}^{*}\left(t_{1}\right)=0$ and $v_{2}^{*}(t)= \pm v_{m}$ for $t>t_{1}$. Since $F_{n}$ is continuously differentiable, and $I_{2}^{*}$ is continuous, $v_{2}^{*}$ must also be continuous at $t=t_{1}$. Therefore, $v_{2}^{*}\left(t_{1}^{-}\right)=v_{2}^{*}\left(t_{1}^{+}\right)= \pm v_{m}$.

We next consider the more general case that the signals are switched from Type A to Type C at $t=t_{1}$, when $I_{1}^{*}$ starts to increase at the maximum rate.

1) We use the signals in Fig. 4 to illustrate our proof. For $t \in\left[0, t_{1}\right], I_{1}^{*}=0$ and at $t=t_{1}, I_{1}^{*}$ starts to increase under $v_{1}^{*}=v_{m}$ (not $-v_{m}$ since it is assumed that $I_{1}^{*} \geq 0$ ). Suppose on the contrary that the signals are switched to Type $\mathrm{D}$ at $t=t_{2}$ and $\left|v_{2}^{*}(t)\right|<v_{m}, I_{2}^{*}(t)>0$ for all $t \in\left[t_{1}, t_{2}\right]$. Then, after $t_{2}, v_{2}^{*}= \pm v_{m}$. Hence, there is a jump of $v_{2}^{*}$ at $t_{2}$. Since $I_{1}^{*}\left(t_{2}\right)>$ $0, I_{2}^{*}\left(t_{2}\right)>0$ and the currents are continuous, it follows from (20) and the continuity of its right-hand side (rhs) that there must also be a jump of $v_{1}^{*}$ at $t_{2}$. Hence, $v_{1}^{*}\left(t_{2}^{+}\right)<v_{m}$.

Let $t_{3}$ be a time instant in this Type $\mathrm{D}$ interval and $t_{3}>t_{2}$. We consider the problem of minimizing the energy over the interval $\left[0, t_{3}\right]$. Generally, the signals that minimizes the energy over a subinterval needn't be the same as the segments of signals that minimize the energy over the entire interval. But if we impose a constraint on the initial and the final values of $I_{1}$ and $I_{2}$ for the subinterval such that they are equal to the corresponding optimal values for the entire interval, then the optimal signal for the subinterval must also be optimal for the entire interval. Here to disjoint $\left[0, t_{3}\right]$ from other subintervals, we only need to impose a constraint on $I_{1}\left(t_{3}\right)$ and $I_{2}\left(t_{3}\right): I_{1}\left(t_{3}\right)=I_{1}^{*}\left(t_{3}\right)$ and $I_{2}\left(t_{3}\right)=I_{2}^{*}\left(t_{3}\right)$. What we will show in the following is that the signals with a switch at $t_{2}$ are not optimal for the subinterval $\left[0, t_{3}\right]$ under the constraint, $I_{1}\left(t_{3}\right)=I_{1}^{*}\left(t_{3}\right)$ and $I_{2}\left(t_{3}\right)=$ $I_{2}^{*}\left(t_{3}\right)$, and hence not optimal for the entire interval. 


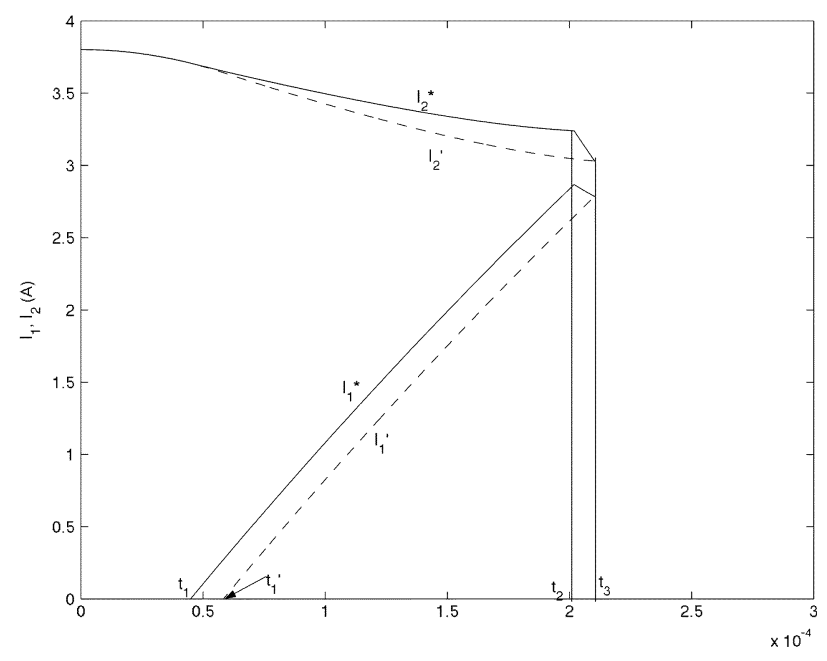

Fig. 4. Illustration for the proof of Theorem 1.

We can choose $t_{3}$ such that $v_{1}^{*}<v_{m}$ on $\left(t_{2}, t_{3}\right]$. If we increase $v_{1}$ to $v_{1}^{\prime}=v_{1}^{*}+\Delta v_{1}, \Delta v_{1}(t)>0$ for $t \in\left(t_{2}, t_{3}\right]$ and keep $I_{1}\left(t_{3}\right)=I_{1}^{*}\left(t_{3}\right)$, then $I_{1}$ will be decreased to $I_{1}^{\prime}$ (see the dashed curve). This can be seen as follows. From

$$
I_{1}^{*}\left(t_{3}\right)=e^{-R\left(t_{3}-t\right) / L_{0}} I_{1}(t)+\frac{1}{L_{0}} \int_{t}^{t_{3}} e^{-R\left(t_{3}-\tau\right) / L_{0}} v_{1}(\tau) d \tau
$$

we have

$$
I_{1}(t)=e^{R\left(t_{3}-t\right) / L_{0}} I_{1}^{*}\left(t_{3}\right)-\frac{1}{L_{0}} \int_{t}^{t_{3}} e^{-R(t-\tau) / L_{0}} v_{1}(\tau) d \tau .
$$

It is obvious that an increase of $v_{1}(t)$ for $t \in\left(t_{2}, t_{3}\right]$ will result in a decrease of $I_{1}$ for $t<t_{3}$. Meanwhile, $t_{1}$ will be increased to $t_{1}^{\prime}$ (see Fig. 4). Let $I_{1}^{\prime}(t)=0$ for $t \in\left[t_{1}, t_{1}^{\prime}\right]$, then $I_{1}^{\prime}(t)<I_{1}^{*}(t)$ for all $t \in\left(t_{1}, t_{3}\right)$. Let $I_{2}^{\prime}$ satisfy $I_{2}^{\prime 2}-I_{1}^{\prime 2}=F_{n} / c_{f}$, then $I_{2}^{\prime}(t)<I_{2}^{*}(t)$ for all $t \in\left(t_{1}, t_{3}\right)$. For $t \in\left[0, t_{1}\right]$, let $I_{1}^{\prime}(t)=$ $I_{1}^{*}(t), I_{2}^{\prime}(t)=I_{2}^{*}(t)$, then the total energy over $\left[0, t_{3}\right]$ for $I_{1}^{\prime}$ and $I_{2}^{\prime}$ is strictly less than that for $I_{1}^{*}$ and $I_{2}^{*}$. This will indicate that the signals $I_{1}^{*}$ and $I_{2}^{*}$ are not optimal if we can ensure that the voltage $v_{2}^{\prime}$ corresponding to $I_{2}^{\prime}$ satisfies the bound $\left|v_{2}^{\prime}\right| \leq v_{m}$ for $t \in\left(t_{1}, t_{3}\right)$.

Since $F_{n}$ is continuously differentiable, since $I_{1}^{*}$ and $I_{2}^{*}$ are continuous and $I_{1}^{*}\left(t_{2}\right), I_{2}^{*}\left(t_{2}\right)>0$, it follows from (20) that if there is a jump decrease of $v_{1}^{*}$ at $t_{2}$, there must also be a jump decrease of $v_{1}^{*}$ at $t_{2}$. This shows that $v_{2}^{*}(t)$ will not take the value $v_{m}$, but instead, it can only be $-v_{m}$ after the switch to Type D.

Let $\Delta v_{1}(t)>0, t \in\left(t_{2}, t_{3}\right)$ be an arbitrarily small increase of $v_{1}$ from $v_{1}^{*}$ and let $\Delta I_{1}, \Delta I_{2}$ and $\Delta v_{2}$ be the corresponding increments of $I_{1}, I_{2}$, and $v_{2}$, respectively. To keep the rhs of (20) unchanged, we must have

$$
\Delta I_{2} v_{2}^{*}+I_{2}^{*} \Delta v_{2}-\Delta I_{1} v_{1}^{*}-I_{1}^{*} \Delta v_{1}=o\left(\left\|\Delta v_{1}\right\|\right)
$$

where $\left\|\Delta v_{1}\right\|=\max \left\{\left|\Delta v_{1}(t)\right|, t \in\left(t_{2}, t_{3}\right]\right\}$. Suppose that $t_{3}-t_{2}$ is arbitrarily small, then for $t \in\left(t_{2}, t_{3}\right),\left|\Delta I_{1}(t)\right| \leq$ $O\left(\left(t_{3}-t_{2}\right)\left\|\Delta v_{1}\right\|\right)$ and $\left|\Delta I_{2}(t)\right| \leq O\left(\left(t_{3}-t_{2}\right)\left\|\Delta v_{2}\right\|\right)$. Hence $\Delta v_{2}(t) \approx\left(I_{1}^{*}(t) / I_{2}^{*}(t)\right) \Delta v_{1}(t)>0$ for $t \in\left(t_{2}, t_{3}\right)$. Since $v_{2}^{*}=-v_{m}$, we have $\left|v_{2}^{\prime}(t)\right|<v_{m}$ for $t \in\left(t_{2}, t_{3}\right)$. As to the subinterval $\left[t_{1}, t_{2}\right]$, let $\Delta v_{1}(t)=0$ on this interval. Since $\Delta I_{1}$ is arbitrarily small, $\Delta I_{2}$ is also arbitrarily small. Hence, from (21), we know that $\Delta v_{2}$ is arbitrarily small. Recalling that $\left|v_{2}^{*}(t)\right|<v_{m}$, we have $\left|v_{2}^{\prime}(t)\right|<v_{m}$ in this interval $\left[t_{1}, t_{2}\right]$.

In view of these arguments, the energy over $\left[0, t_{3}\right]$ can be further reduced without violating the constraint on the voltages. This indicates that the signals with a switch to Type D before $\left|v_{2}^{*}\right|$ reaches $v_{m}$ or $I_{2}^{*}$ reaches 0 is not optimal. This completes the proof of 1).

2) This can be proven similarly to 1) by showing that the energy can be further reduced by increasing $v_{1}$ in a small interval after $t_{2}$.

3) This is obvious since $I_{1}^{*}$ and $I_{2}^{*}$ are continuous signals.

Similarly, we have the following theorem.

Theorem 2: Let $\left(I_{1}^{*}, I_{2}^{*}, v_{1}^{*}, v_{2}^{*}\right)$ be a set of optimal signals. Suppose that the first interval is of Type B $\left(I_{1}^{*}>0, I_{2}^{*}=0\right)$. The signals can switch to Type A $\left(I_{1}^{*}=0, I_{2}^{*}>0\right)$ at $t=t_{1}$ only if $F_{n}\left(t_{1}\right)=0$. They can switch to Type $\mathrm{C}\left(\left|v_{2}^{*}\right|<v_{m}, v_{1}^{*}=\right.$ $\left.\pm v_{m}\right)$ at $t=t_{1}$ only if $v_{1}^{*}\left(t_{1}^{-}\right)= \pm v_{m}$. Consider the case that they switch to Type $\mathrm{D}$ (with $v_{2}^{*}=v_{m},\left|v_{1}^{*}\right|<v_{m}$ ) at $t=t_{1}$. Assume that $\left|v_{1}^{*}\left(t_{1}\right)\right|<v_{m}$. Then, the signals will not switch to:

1) Type $\mathrm{C}$ before $\left|v_{1}^{*}\right|$ reaches $v_{m}$ or before $I_{1}^{*}$ reaches 0 ;

2) Type $\mathrm{D}$ (with $v_{2}^{*}=-v_{m},\left|v_{1}^{*}\right|<v_{m}$ ) before $\left|v_{1}^{*}\right|$ reaches $v_{m}$ or before $I_{1}^{*}$ reaches 0

3) Type A before $I_{1}^{*}$ reaches 0 .

From Theorems 1 and 2, we conclude that, after the signals have switched from Type A to Type C (or from Type B to Type D), there will be no more switch before $\left|v_{1}\right|=\left|v_{2}\right|=v_{m}$ or before one of the currents reaches 0 .

\section{The Optimal Solution: An Example}

In this section, we use an example to illustrate how to obtain the optimal solution using the stationary condition and Theorems 1 and 2 .

Example 2: Consider the same circuit systems as in Example 1. Suppose that $F_{n}(t)=2 \sin (7000 t+\pi / 2)$. Since the signal is periodic and symmetric, we only need to consider a half period. Here we have $T=\pi / 7000=4.4880 \times 10^{-4}$. Let us first plot the optimal signals without considering the voltage constraint in Fig. 5. The actual $v_{1}$ and $v_{2}$ are unbounded but we clipped off from above $v=20$ and below $v=-20$. Between the interval $\left[t_{a}, t_{b}\right]$, either $v_{1}$ or $v_{2}$ exceeds the constraint.

It is clear that Type A or Type B intervals (either $I_{1} \equiv 0$ or $\left.I_{2} \equiv 0\right)$ must be outside of $\left[t_{a}, t_{b}\right]$ and Type A $\left(I_{1} \equiv 0\right)$ can only occur between 0 and $t_{a}$ and Type $\mathrm{B}\left(I_{2} \equiv 0\right)$ can only occur between $t_{b}$ and $T$. We assume that the first interval is Type A.

It is also obvious that the second interval cannot be of Type B since $\left[t_{a}, t_{b}\right]$ must be covered with Type $\mathrm{C}$ or Type $\mathrm{D}$ intervals. We first try the second interval with Type $\mathrm{D}\left(v_{2}= \pm v_{m},\left|v_{1}\right|<\right.$ $\left.v_{m}\right)$. By Theorem 1, the switching time must be at $t_{1}=t_{a}$ when $v_{2}$ reaches $-10 \mathrm{~V}$. Computation reveals that this switch to Type $\mathrm{D}$ will result in $v_{1}>10$ for $t>t_{1}$. Hence, we can exclude this possibility and try to switch to Type $\mathrm{C}$ at certain $t_{1}$. By Theorem 1, there will be no switch to other types after $t_{1}$ and before $\left|v_{2}\right|=v_{m}$ or $I_{2}=0$. With different $t_{1}$, we obtained three outcomes. 

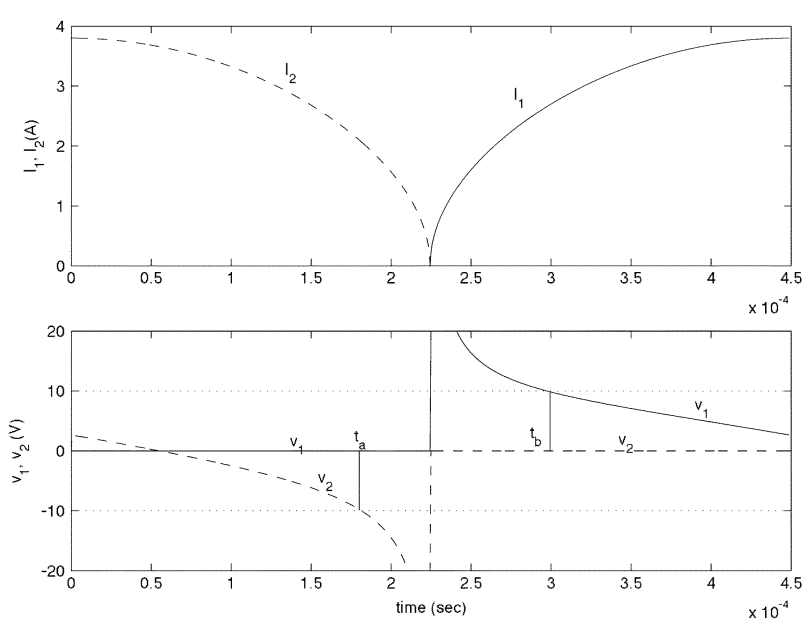

Fig. 5. Optimal signals without considering the voltage constraint

1) $t_{1}>1.5313$. The signal $v_{2}(t)$ will reach $-v_{m}=-10$ $\mathrm{V}$ at some $t_{2}>t_{1}$, where $I_{1}\left(t_{2}\right), I_{2}\left(t_{2}\right)>0$. Clearly, it is impossible to switch to Type A or B at $t_{2}$. The switch to Type $\mathrm{D}$ at $t_{2}$ will cause $v_{1}$ to exceed the bound (see Fig. 6, the upper small figure). Also, if there is no switch at $t_{2}, v_{2}$ will exceed the bound after $t_{2}$. Hence, this case is infeasible.

2) $t_{1}<1.5313$. The signal $v_{2}$ will not reach $-v_{m}$ and $I_{2}$ will not reach 0 before $v_{1}\left(t_{2}\right)=v_{2}\left(t_{2}\right)=v_{m}$ at some $t_{2}$ (see Fig. 6, bottom). At $t_{2}, I_{1}\left(t_{2}\right), I_{2}\left(t_{2}\right)>0$. No matter which type is switched at $t=t_{2}$ or not switch, either $v_{1}$ or $v_{2}$ will go out of the bound after $t_{2}$. Hence, this case is also infeasible.

3) $t_{1}=1.5313$. The signal $v_{2}$ will not reach $-v_{m}$ before $I_{2}$ reaches 0 at some $t_{2}$. The switch to Type B at $t_{2}$ results in feasible solution and Type $\mathrm{B}$ can be continued until $t=T$ (see Fig. 7).

We see that the third case is the only solution, among other possible optimal solutions, that are feasible for the interval $\left[0, t_{2}^{+}\right)$. After $t_{2}$, switching to any other type will increase the energy consumption. Hence it is the optimal solution. Fig. 7 plots the optimal currents and voltages, where the dashed curves in the current figure are the optimal currents for the case of no bound on the voltages. Fig. 8 plots the auxiliary signals $\alpha_{1}^{*}, \alpha_{2}^{*}$, and $\lambda^{*}$. Note that $\lambda^{*}$ has an impulse at the instant of the second switch. The minimal energy over the half period $[0, T]$ is found to be $E=4.3175 c_{e} \times 10^{-3}$.

It can be expected that the optimal signals for all the sinusoidal force signals $F_{n}(t)$ will have the same pattern as demonstrated in Example 2, which completely depends on the first switching time $t_{1}$. It turns out that the signals are very sensitive to $t_{1}$. A small variation of $t_{1}$ from the optimal value will cause nonfeasibility, not just nonoptimality, of the resulting signals. We can also expect that an optimal $t_{1}$ for a certain force signal will cause nonfeasibility when the force signal has a tiny change in magnitude or frequency. Because of this high sensitivity of the optimal solution, we would like to find some static relations $F_{n} \mapsto I_{1}$ and $F_{n} \mapsto I_{2}$ so that the energy consumption is close to the minimal value achieved by the open loop design.
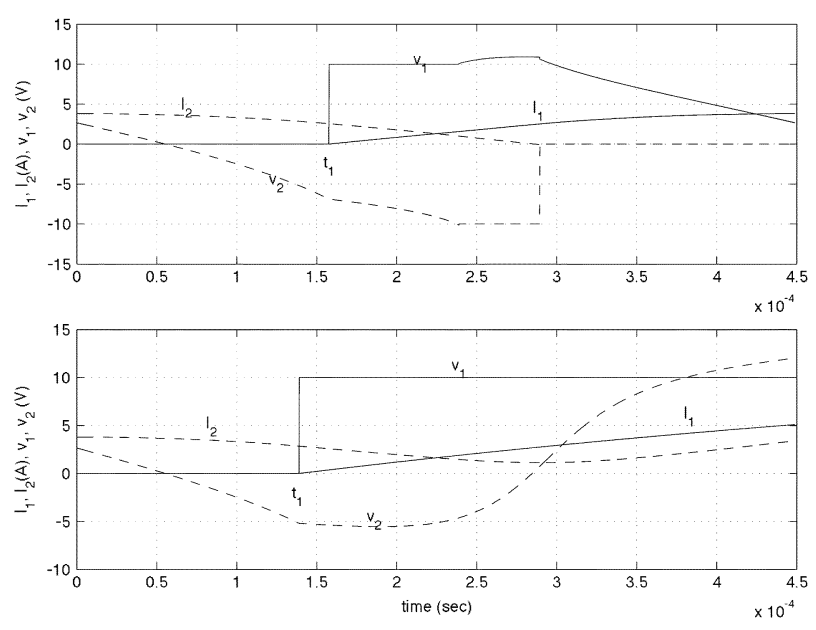

Fig. 6. Some nonfeasible solutions resulting from different $t_{1}$.
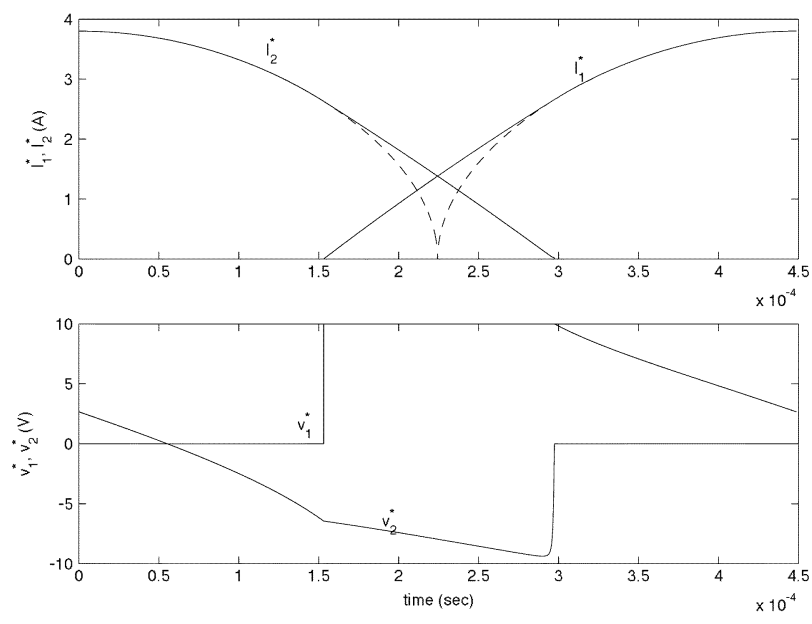

Fig. 7. Optimal allocation and the signals.

\section{Static Allocation Strategies}

In this section, we will consider static allocation and closed-loop circuit systems. We will again use $I_{1 d}, I_{2 d}$, and $F_{n d}$ to denote the desired current and force signals.

\section{A. A Class of Simple Allocation Functions}

By static allocation, we mean that the values of $I_{1 d}(t)$ and $I_{2 d}(t)$ only depend on $F_{n d}(t)$ for any $t$. We consider the following class of simple allocation functions with a design parameter $F_{0}$ :

and

$$
I_{1 d}= \begin{cases}0, & \text { if } F_{n d}>F_{0} \\ \frac{F_{0}-F_{n d}}{2\left(F_{0} c_{f}\right)^{\frac{1}{2}},} & \text { if }\left|F_{n d}\right| \leq F_{0} \\ \left(-F_{n d} / c_{f}\right)^{\frac{1}{2}}, & \text { if } F_{n d}<-F_{0}\end{cases}
$$

$$
I_{2 d}=\left\{\begin{array}{ll}
0, & \text { if } F_{n d}<-F_{0} \\
\frac{F_{0}+F_{n d}}{2\left(F_{0} c_{f}\right)^{\frac{1}{2}},} & \text { if }\left|F_{n d}\right| \leq F_{0} \\
\left(F_{n d} / c_{f}\right)^{\frac{1}{2}}, & \text { if } F_{n d}>F_{0}
\end{array} .\right.
$$

Fig. 9 plots some functions from this class with $c_{f}=0.1384$ and $F_{0}=0,0.5,1,1.5$, and 2, respectively. For $\left|F_{n d}\right| \leq F_{0}$, the currents are affine in $F_{n d}$, and for $\left|F_{n d}\right|>F_{0}$, the currents are square roots of $\left|F_{n d}\right| / c_{f}$ or 0 . If $F_{n d}$ is sinusoidal and its 

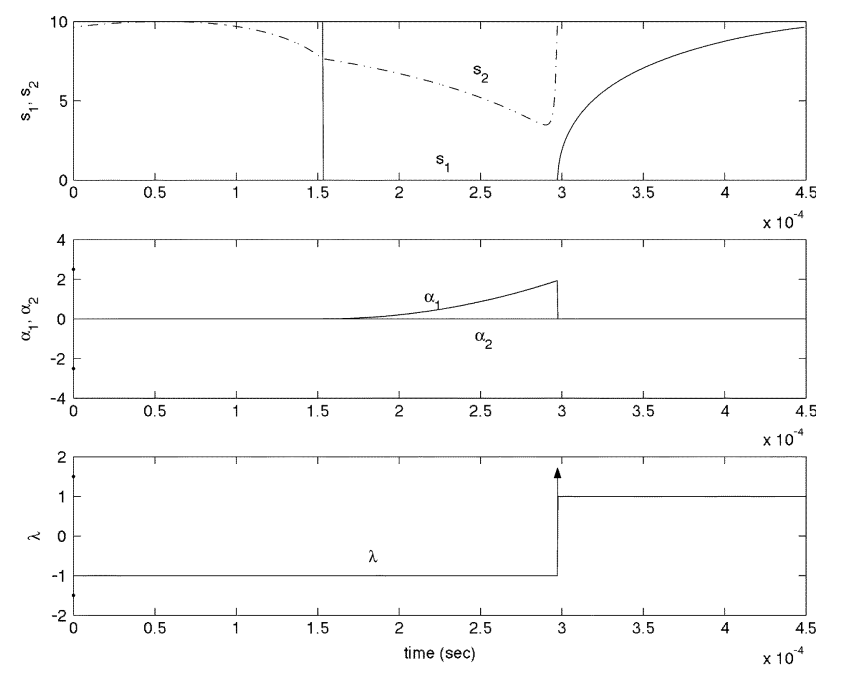

Fig. 8. Auxiliary optimal signals.

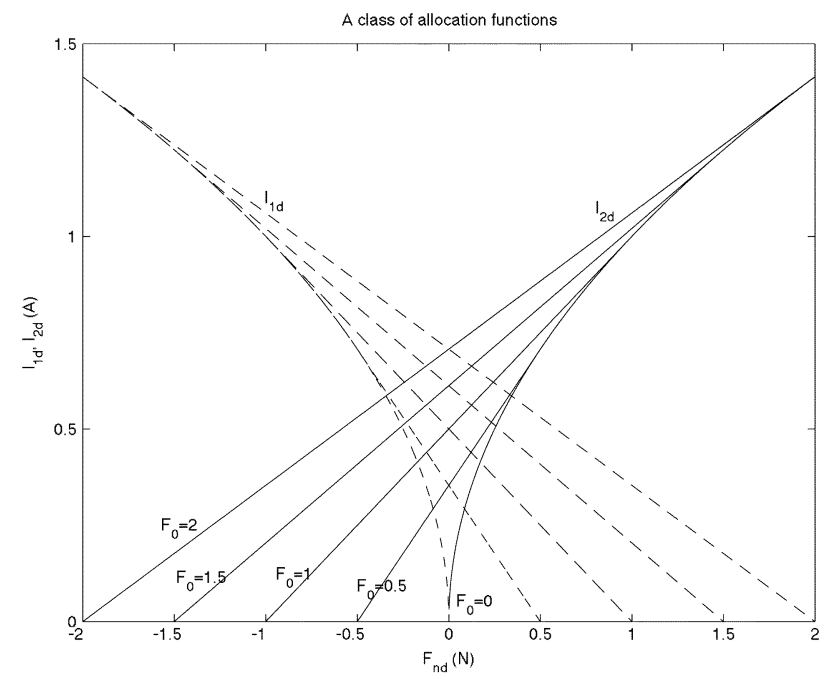

Fig. 9. A class of allocation functions.

magnitude is $F_{0}$, then $I_{1 d}$ and $I_{2 d}$ will both be sinusoidal and the functions in (22) and (23) result in full bias allocation. If $F_{0}=0$, then (22) and (23) result in single actuator allocation. Let $I_{b}=$ $\left(F_{0} / c_{f}\right)^{(1 / 2)} / 2$, then for $F_{n d}=0$, we have $I_{1 d}=I_{2 d}=I_{b}$. We also call this $I_{b}$ the current bias. It is clear that smaller $F_{0}$ will result in smaller current bias and reduced power loss. The following example will show that a suitable static allocation can reduce the power loss to a level very close to that by the optimal nonstatic allocation.

Example 3: We consider the same circuit systems and force signal as in Example 2, $F_{n d}(t)=2 \sin (7000 t+\pi / 2)$. We use static allocation functions from the class in (22) and (23) to determine $I_{1 d}(t)$ and $I_{2 d}(t)$. The voltages are determined from

$$
v_{1}=L_{0} \dot{I}_{1 d}+R I_{1 d}, \quad v_{2}=L_{0} \dot{I}_{2 d}+R I_{2 d}
$$

This is possible if all the signals are determined off-line (openloop). The allocation function from this class that results in the least energy consumption while keeping the voltages bounded by $10 \mathrm{~V}$ is found to be the one with $F_{0}=1.095$. Under this allocation function, the energy consumption in a half period is $E=4.3379 c_{e} \times 10^{-3}$, slightly greater than the optimal value
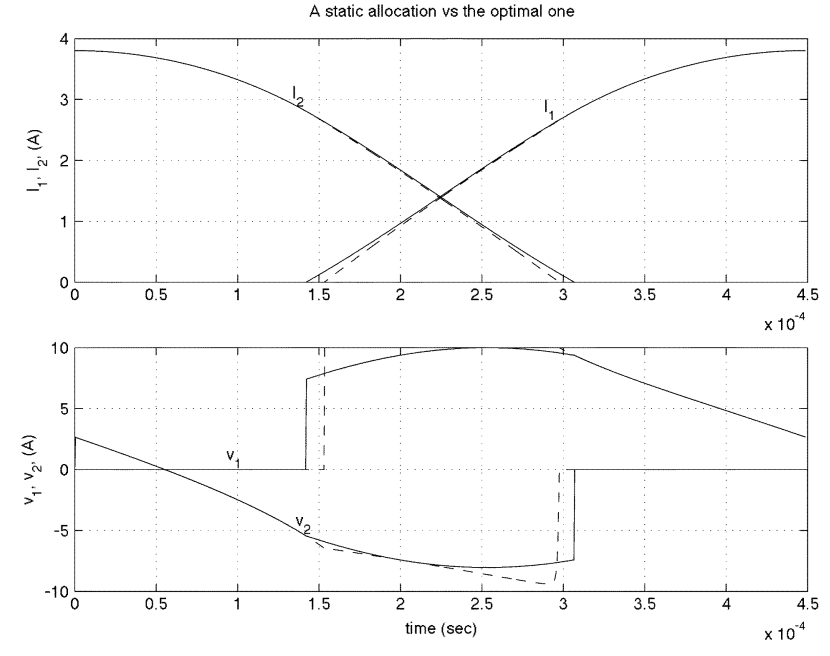

Fig. 10. Static allocation as compared with the optimal one.

$4.3175 c_{e} \times 10^{-3}$. In Fig. 10, the current signals and the voltage signals under this static allocation are plotted as compared to the optimal signals (in dashed curves). We see that the two sets of signals are very close.

This example shows that a static allocation can achieve almost the same minimal energy consumption as that by the optimal (nonstatic) allocation. Here, we should note that the situation in Example 3 is still not a closed-loop type. It is not practical to generate $v_{1}$ and $v_{2}$ from (24) on-line.

As we have discussed in Section II, there are two parts in a closed-loop design. Apart from allocating the currents statically, we also need to design feedback controls $v_{1}=g_{1}\left(I_{1}, I_{1 d}\right)$ and $I_{2}=g_{2}\left(I_{2}, I_{2 d}\right)$ such that $I_{1}$ and $I_{2}$ will closely follow $I_{1 d}$ and $I_{2 d}$. One way is to first search for an allocation function, then choose feedback laws for the circuit systems. The second way is to first fix the feedback laws and then choose a proper allocation strategy. Here, we take the second way for example.

Suppose that we are given the circuit control laws as $v_{1}=$ $k\left(I_{1}-I_{1 d}\right)$ and $v_{2}=k\left(I_{2}-I_{2 d}\right)$. We can choose the smallest $F_{0}$ that satisfy certain tracking performance by using frequency analysis. It turns out that by increasing $F_{0}$, the higher frequency components of the signal $I_{1 d}$ can be reduced and hence the voltage saturation can be avoided, resulting in better tracking performance. The following table compares the Fourier coefficients of $I_{1 d}$ for different $F_{0} \mathrm{~s}$ (with $F_{n d}=2 \sin (7000 t)$ ). The last column in the table shows the energy consumption, where $K=c_{e} \times 10^{-3}$ :

\begin{tabular}{c|c|c|c|c|c}
\hline$F_{0}$ & $c_{0}$ & $c_{1}$ & $c_{3}$ & $c_{5}$ & $E$ \\
\hline \hline 0 & 0.3389 & 0.2472 & 0.0353 & 0.0161 & $4 \mathrm{~K}$ \\
\hline 0.5 & 0.3510 & 0.2466 & 0.0336 & 0.0133 & $4.0426 \mathrm{~K}$ \\
\hline 1.0 & 0.3732 & 0.2437 & 0.0260 & 0.0040 & $4.1706 \mathrm{~K}$ \\
\hline 1.5 & 0.4035 & 0.2369 & 0.0126 & -0.0039 & $4.3897 \mathrm{~K}$ \\
\hline 2.0 & 0.4448 & 0.2221 & 0 & 0 & $4.7185 \mathrm{~K}$ \\
\hline
\end{tabular}

From the table, we see that $c_{3}$ and $c_{5}$ are much smaller for $F_{0}=$ 1 than for $F_{0}=0.5$ and the increase of total energy is not obvious. This suggests that a good tracking performance could be maintained by taking $F_{0} \approx 1$ while the energy consumption is significantly reduced. However, since the relation between the Fourier coefficients and the magnitude of the voltage is hard to 
characterize, it would be straightforward to find the minimal $F_{0}$ which would avoid voltage saturation by simulation.

Example 4: Consider the same circuit systems as in Example 1 and $F_{n d}(t)=2 \sin (7000 t)$. The circuit control laws are $v_{1}=$ $50\left(I_{1 d}-I_{1}\right)$ and $v_{2}=50\left(I_{2 d}-I_{2}\right)$. The minimal $F_{0}$ that will avoid voltage saturation is found to be $F_{0}=1.05$, much smaller than the magnitude of $F_{n d}$. Fig. 11 illustrates the tracking performances under different $F_{0}$. In the first column, $F_{0}=0$, where the single actuator allocation strategy is used. In the third column, $F_{0}=2$, where a full bias allocation strategy is used. The second column shows the tracking performance by using $F_{0}=1.05$. As we can see, the tracking performance is almost the same as that in the third column. This shows that the tracking performance can be well maintained by using a much smaller biasing level. Since the power loss is proportional to $I_{1}^{2}+I_{2}^{2}$, and $I_{2}$ has the same shape with $I_{1}$, we see that the power loss is much smaller than the case where $F_{0}=2$. Exact computation shows that the energy consumption (during a half period in the steady state) corresponding to $F_{0}=2,1.05,0$, are respectively, $4.7299 c_{e} \times 10^{-3}, 4.1958 c_{e} \times 10^{-3}$, and $3.3963 c_{e} \times 10^{-3}$. Here, we note that the energy consumption for $F_{0}=1.05$ is less than the minimal value by open-loop design. This is not a contradiction since there is a tracking error between $I_{1}$ and $I_{1 d}$. Actually, if we compute the energy from $I_{1 d}$ instead of $I_{1}$, then the value is $4.3247 c_{e} \times 10^{-3}$.

Finally, we note that the minimal value of $F_{0}$ for the full closed-loop design is 1.05 , which is very close to the minimal value of $F_{0}=1.095$ under the assumption of no tracking error.

As can be seen from optimization problem (5), the optimal allocation strategy depends on the desired force signal. An allocation strategy cannot be optimal for all the signals. Since the steady state consumes most of the energy, we may use the steady state desired net force signal for optimization. For system (1), $F_{n d}(t)=-d(t)$ in the steady state.

Given $F_{n d}$, a guideline for choosing a static allocation from (22) and (23) is to determine the smallest number $F_{0}$ such that voltage saturation is avoided. It is clear that such an allocation will also avoid voltage saturation if the magnitude of the desired force is decreased or its frequency is decreased. To guarantee a satisfactory force tracking performance, we need to choose the force signal which has the largest magnitude and the highest frequency.

\section{B. Other Possible Allocation Functions}

The allocation functions in (22) and (23) are not differentiable at $F_{n d}= \pm F_{0}$. This can be avoided by modifying them around $F_{n d}= \pm F_{0}$, as illustrated in Fig. 12, where $I_{2 d}$ is replaced with $k_{1}\left(1-\cos \left(\left(F_{n d} \pm\left(F_{0}+e\right)\right)\right)\right.$ in a neighborhood of $F_{0}$. In the case of $F_{0}=1.05$, we choose $k_{1}=0.88, e=0.3$.

The following is a comparison of the Fourier coefficients and the total energy between the modified and the unmodified allocation function:

\begin{tabular}{c|c|c|c|c|c}
\hline & $c_{0}$ & $c_{1}$ & $c_{3}$ & $c_{5}$ & $E$ \\
\hline \hline Mod & 0.3770 & 0.2427 & 0.0239 & 0.0025 & $4.1989 \mathrm{~K}$ \\
\hline Unmod & 0.3759 & 0.2433 & 0.0249 & 0.0029 & $4.1958 \mathrm{~K}$ \\
\hline
\end{tabular}
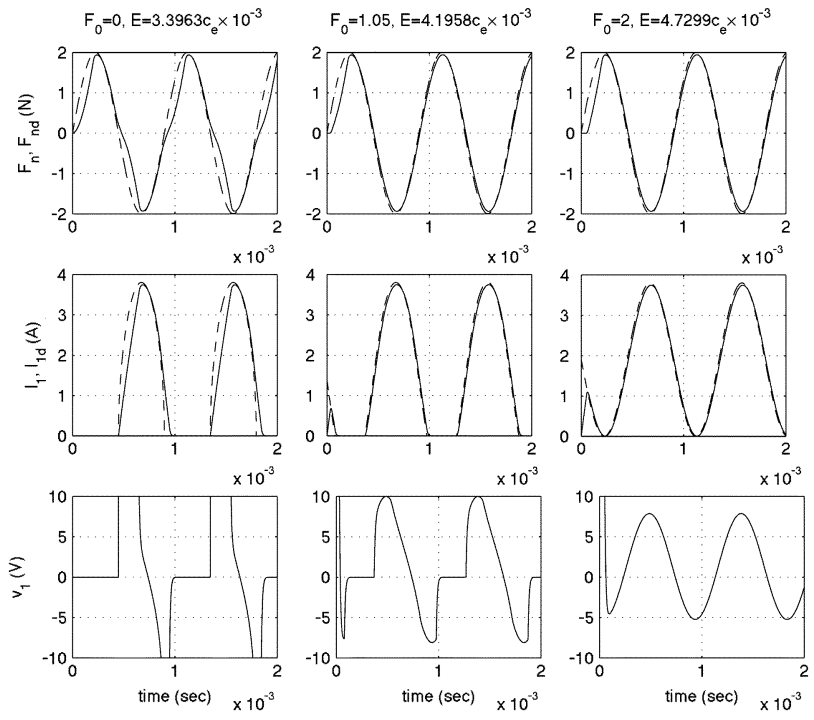

Fig. 11. Tracking performances with respect to different current allocation.

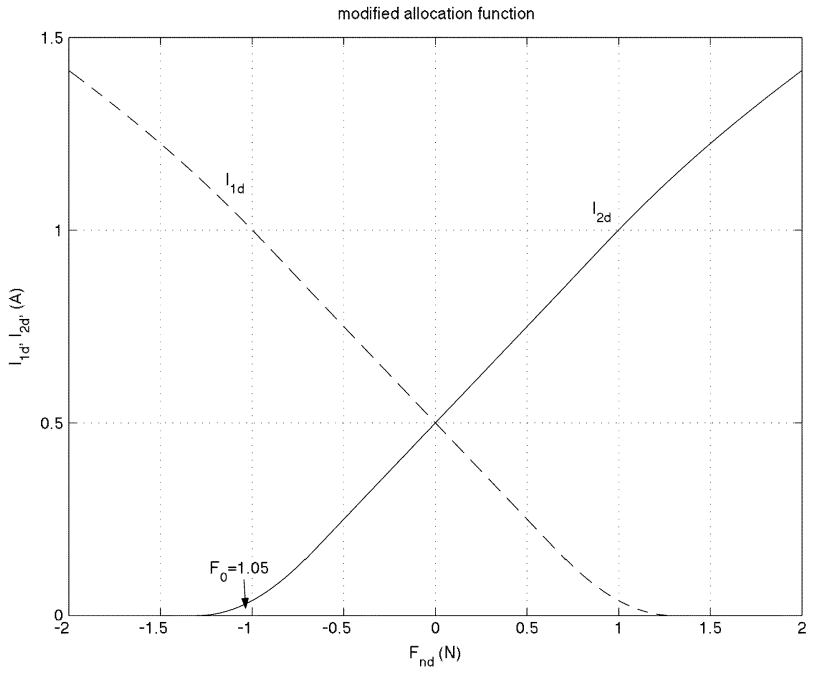

Fig. 12. A modified allocation function.

As we can see from the table, the difference in the coefficients and the energy is very small between the modified and unmodified allocation functions.

There are many other choices of the allocation functions. The principle is to keep energy consumption low while voltage saturation is avoided.

\section{CONCLUSION}

Power loss reduction and performance improvement are conflicting objectives. In this paper, we investigated the fundamental reason behind performance degradation when single actuator allocation strategy is adopted. Based on our investigation results, we formulated the problem of minimizing power loss under the constraint of bounded voltages. Optimal solution was obtained for the class of sinusoidal force signals. We also presented some static allocation strategies that would result in a suboptimal power loss. 


\section{REFERENCES}

[1] CRC Handbook of Lubrication and Tribology, vol. 3, R. Booser, Ed., CRC, Boca Raton, FL.

[2] M. Baloh, "Practical concerns of linearizing AMB: Power loss reduction, amplifier dynamics, and flux estimation," in Proc. 6th Int. Symp. Magnetic Suspension Technology (ISMST), Turin, Italy, Oct. 2001, pp. $105-110$.

[3] — , "Time varying feedback linearization of magnetic bearing actuator," Ph.D. dissertation, Univ. Virginia, Charlottesville, 2001.

[4] A. Chara, "Nonlinear control of a magnetic levitation system without premagnetization," IEEE Trans. Control Syst. Technol., vol. 4, pp. 513-523, Sept. 1996.

[5] T. Hu, Z. Lin, B. Huang, and P. E. Allarie, "On minimum current biasing and control for a balanced beam suspended on magnetic bearings," in Proc. 6th Int. Symp. Magnetic Suspension Technology (ISMST), Turin, Italy, Oct. 2001, pp. 410-415.

[6] D. Johnson, G. V. Brown, and D. I. Inman, "Adaptive variable bias magnetic bearing control," in Proc. 1998 Amer. Control Conf., Philadelphia, PA, 1998.

[7] J. Levine, J. Lottin, and J.-C. Ponsart, "A nonlinear approach to the control of magnetic bearings," IEEE Trans. Control Syst. Technol., vol. 4 pp. 524-544, Sept. 1996.

[8] L. Li, "Linearization of feedback actuators by constant current sum, constant voltage sum and constant flux sum," IEEE Trans. Magn., vol. 35, pp. 528-535, Jan. 1999.

[9] L. Li and J. Mao, "Feedback linearization of magnetic bearing actuators for uniform upward bound of force slew rate," Proc. Inst. Elect. Eng.-Elect. Power Applicat., vol. 146, no. 4, 1999.

[10] F. Lösch, "Two remarks on the modeling of active magnetic bearing systems," in Proc. 6th Int. Symp. Magnetic Suspension Technology (ISMST), Turin, Italy, Oct. 2001, pp. 422-427.

[11] E. H. Malsen, P. E. Allaire, M. D. Noh, and C. K. Sortore, "Magnetic bearing design for reduced power consumption," J. Tribology, vol. 118, July 1996.

[12] C. Yang, "Control of magnetic bearings with low bias flux and saturating voltage," M.S. thesis, Univ. Virginia, Charlottesville, 1997.

Tingshu Hu (S'99-SM'01) received the B.S. and M.S. degrees in electrical engineering from Shanghai Jiao Tong University, Shanghai, China, and the Ph.D. degree in electrical engineering from the University of Virginia, Charlottesville, in 2001

She is currently a Research Associate with the Department of Electrical and Computer Engineering, University of Virginia. Her research interests include nonlinear systems theory, optimization, robust control theory, and control application in mechatronic systems and biomechanical systems. She is a coauthor (with Zongli Lin) of the book Control Systems with Actuator Saturation: Analysis and Design (Boston, MA: Birkhäuser, 2001).

Dr. Hu is an Associate Editor on the Conference Editorial Board of the IEEE Control Systems Society.
Zongli Lin (S'92-M'93-SM'98) received the B.S. degree in mathematics and computer science from Xiamen University, Xiamen, China, in 1983, the M.Eng. degree in automatic control from Chinese Academy of Space Technology, Beijing, China, in 1989, and the Ph.D. degree in electrical and computer engineering from Washington State University, Pullman, in 1994

$\mathrm{He}$ is currently an Associate Professor with the Department of Electrical and Computer Engineering, University of Virginia, Charlottesville. Previously, he was a Control Engineer at the Chinese Academy of Space Technology and an Assistant Professor with the Department of Applied Mathematics and Statistics, State University of New York at Stony Brook. His current research interests include nonlinear control, robust control, and modeling and control of magnetic bearing systems. In these areas he has published several papers. He is also the author of the book Low Gain Feedback (London, U.K.: Springer-Verlag, 1998) and a coauthor (with Tingshu Hu) of the recent book Control Systems with Actuator Saturation: Analysis and Design (Boston, MA: Birkhäuser, 2001).

Dr. Lin served as an Associate Editor of IEEE TRANSACTIONS ON AUTOMATIC CONTROL from 2001 to 2003 . He is currently a member of the IEEE Control Systems Society's Technical Committee on Nonlinear Systems and Control and heads its Working Group on Control with Constraints. He is the recipient of a U.S. Office of Naval Research Young Investigator Award.

Paul E. Allaire received the B.S. and M.E. degrees from Yale University, New Haven, CT, and the Ph.D. degree in mechanical engineering from Northwestern University, Evanston, IL, in 1971.

He has been a faculty member at the University of Virginia, Charlottesville, since 1972. Currently, he is the Wade Professor of Engineering in the Department of Mechanical and Aerospace Engineering, University of Virginia. His research interests span several areas of rotating machinery: bearings, rotor dynamics, seals, controls, and fluid flows. His primary research interest at this time is in magnetic bearings, including bearing design, shaft dynamics, and controls. $\mathrm{He}$ is also active in biomechanics research. He has published over 140 technical publications including over 50 refereed journal publications and one textbook on finite elements. He has had extensive funded research programs with government and industry over the past 30 years. A major research activity is the design, development, and testing of a magnetic bearing supported artificial heart for human implantation. Several prototypes have been successfully testing in pumping both water and blood. Much research activity is underway to improve the magnetic bearing designs, enhance control system design using robust control methods, real-time computer controls, development of self sensing algorithms, design of more efficient pumps, computational fluids analysis of the flow in the heart pump, and other aspects. He is the Director of the Virginia Artificial Heart Research Program. The project is in collaboration with the Utah Artificial Heart Laboratory and Medquest Products, Inc. 